\title{
Study of Thorium-Plutonium Fuel for Possible Operating Cycle Extension in PWRs
}

\author{
Klara Insulander Björk, ${ }^{1,2}$ Cheuk Wah Lau, ${ }^{2}$ Henrik Nylén, ${ }^{3}$ and Urban Sandberg ${ }^{3}$ \\ ${ }^{1}$ Thor Energy AS, Sommerrogaten 13-15, 0255 Oslo, Norway \\ ${ }^{2}$ Division of Nuclear Engineering, Department of Applied Physics, Chalmers University of Technology, 41296 Gothenburg, Sweden \\ ${ }^{3}$ Ringhals AB, 43285 Väröbacka, Sweden
}

Correspondence should be addressed to Klara Insulander Björk; klara.insulander@scatec.no

Received 14 October 2012; Accepted 11 January 2013

Academic Editor: Hangbok Choi

Copyright (C) 2013 Klara Insulander Björk et al. This is an open access article distributed under the Creative Commons Attribution License, which permits unrestricted use, distribution, and reproduction in any medium, provided the original work is properly cited.

\begin{abstract}
Computer simulations have been carried out to investigate the possibility of extending operating cycle length in the Pressurised Water Reactor Ringhals 3 by the use of thorium-plutonium oxide fuel. The calculations have been carried out using tools and methods that are normally employed for reload design and safety evaluation in Ringhals 3. The 3-batch reload scheme and the power level have been kept unchanged, and a normal uranium oxide fuel assembly designed for a 12-month operating cycle in this reactor is used as a reference. The use of plutonium as the fissile component reduces the worth of control rods and soluble boron, which makes it necessary to modify the control systems. The delayed neutron fraction is low compared with the reference, but simulations and qualitative assessments of relevant transients indicate that the reactor could still be operated safely. Differences in reactivity coefficients are mainly beneficial for the outcome of transient simulations for the thorium based fuel. A $50 \%$ extension of the current 12-month operating cycle length should be possible with thorium-plutonium mixed oxide fuel, given an upgrade of the control systems. More detailed simulations have to be carried out for some transients in order to confirm the qualitative reasoning presented.
\end{abstract}

\section{Introduction}

The objective of the work described herein is to investigate the possibility of extending operating cycle length in a Pressurized Water Reactor (PWR) by the use of ThoriumPlutonium Mixed Oxide (Th-MOX) fuel.

The general viability of Th-MOX fuel in light water reactors (mainly PWRs) has been confirmed by several recent studies [1-5]. In addition, the neutronic and physical properties of Th-MOX indicate that the fuel is not only viable, but may also improve the economy of a nuclear power plant by allowing for longer operating cycles and hence a higher availability of the reactor. Most importantly, the good material properties $[6,7]$ of the Thorium-Plutonium Mixed Oxide ceramic indicate that Th-MOX fuel may be capable of sustaining higher burnups than Uranium-Oxide- (UOX-) based fuel types. Secondly, the currently practiced uranium enrichment limit of 5\% U-235 does not affect Th-MOX, which can be loaded with high amounts of plutonium. Finally, the slow change of the multiplication factor with depletion of Th-MOX makes it possible to achieve high burnups without having an excessively high initial multiplication factor [4].

In this paper, we present and discuss the simulation of a standard PWR fuel assembly loaded with Th-MOX fuel and a full Reload Safety Evaluation (RSE) of the Ringhals 3 PWR core when loaded with this fuel assembly. The content of $\mathrm{Pu}$ and Burnable Absorbers (BA) in the fuel is adapted for achieving an 18-month cycle, which is an operating cycle extension of 50\% compared with the normal cycle length in Ringhals 3 . The fuel assembly design is developed using the fuel assembly burnup simulation program CASMO-4E [8], and the RSE is carried out with SIMULATE-3 [9].

The simulated fuel and reactor systems are described in Section 2 and the calculation tools and methods used in this study in Section 3. The results in terms of depletion behaviour 
Reference core

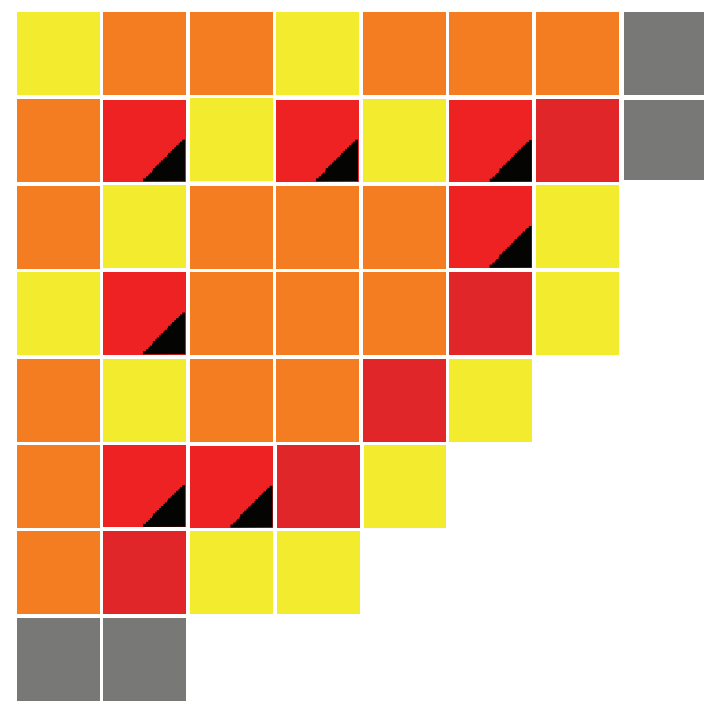

1st cycle with Gd
1st cycle without Gd
2nd cycle

FIgURE 1: The UOX reference core.
Th-Pu core

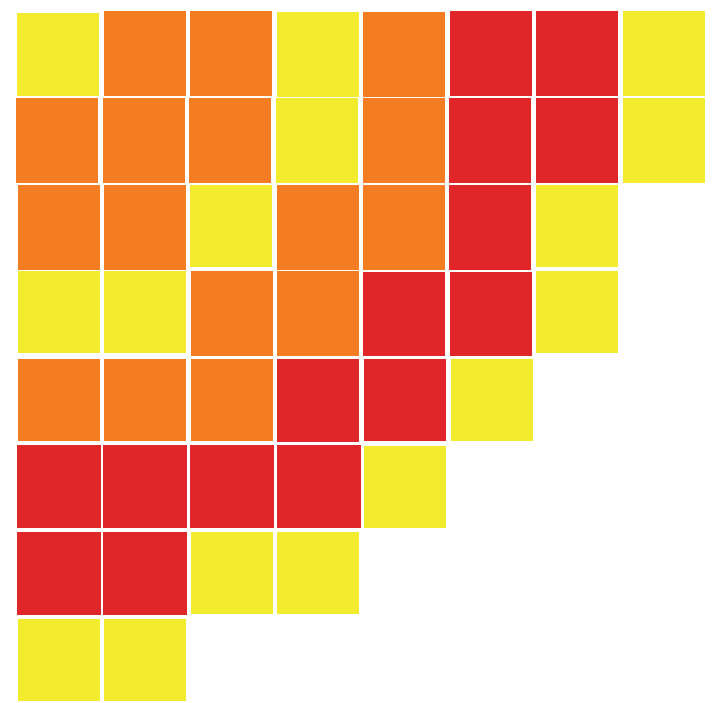

Figure 2: The Th-MOX core.

and neutronic safety parameters are presented in Section 4 and conclusions are drawn in Section 5.

\section{Description of the Modelled System}

2.1. Reactor. The reactor simulated in this study is the Swedish Ringhals 3 reactor, which is a $3135 \mathrm{MW}_{\text {th }}$ reactor of Westinghouse design. This reactor operates with a 12month cycle and a 3-batch reload scheme. In the reference case, which is a typical operation cycle, the Ringhals 3 core is loaded with 28 fuel assemblies containing the $\mathrm{BA} \mathrm{Gd}_{2} \mathrm{O}_{3}$ and 20 fuel assemblies without BA. Such a reload is used as a reference in this work. The core design is shown in Figure 1 and the fuel assemblies will be discussed in more detail in Section 2.2.

The Th-MOX core is designed for an 18-month cycle using a different core layout, designed to reduce the power of the oldest fuel assemblies. Without this change of the layout, the oldest fuel assemblies (having the highest burnup) would run at a power level which would be too high considering the deterioration of the fuel material at high burnup. This core design is shown in Figure 2.

2.2. Fuel Assemblies. The reference fuel is a UOX fuel assembly designed for a standard 12-month cycle in Ringhals 3, which requires an enrichment of $4.4 \%$ in most fuel rods. The assembly comprises 264 fuel rods, arranged in a standard 17by-17 lattice with a central instrumentation thimble and 24 control rod guide tubes. In the assemblies containing $\mathrm{Gd}_{2} \mathrm{O}_{3}$, this is located in 12 rods. Since the addition of $\mathrm{Gd}_{2} \mathrm{O}_{3}$ lowers the thermal conductivity of the fuel, a lower enrichment $(2.8 \%)$ is used in order to avoid high power in these fuel rods. The reference fuel design with $\mathrm{Gd}_{2} \mathrm{O}_{3}$ is shown in Figure 3 . The reference fuel without $\mathrm{BA}$ is identical except that all rods have $4.4 \%$ enrichment and no BA. The fuel assembly heavy metal weight is $461 \mathrm{~kg}$.

The Th-MOX fuel design uses the same mechanical structure as the reference fuel, but differs in two important aspects.

Firstly, the uranium oxide fuel is exchanged for a mixture of thorium and plutonium oxides. The $\mathrm{Pu}$ isotope vector used in the Th-MOX fuel is $2 \%{ }^{238} \mathrm{Pu}, 53 \%{ }^{239} \mathrm{Pu}, 25 \%{ }^{240} \mathrm{Pu}$, $15 \%{ }^{241} \mathrm{Pu}$, and $5 \%{ }^{242} \mathrm{Pu}$. This corresponds to the $\mathrm{Pu}$ vector in spent light water reactor fuel burnt to approximately $42 \mathrm{MWd} / \mathrm{kgHM}$, if reprocessed immediately [10].

Secondly, the Integral Fuel Burnable Absorber (IFBA) concept is preferred over the use of $\mathrm{Gd}_{2} \mathrm{O}_{3}$ as a BA. The reason not to use $\mathrm{Gd}_{2} \mathrm{O}_{3}$ is that it is normally mixed into the fuel matrix. Since Th-MOX is already a mixed oxide, using $\mathrm{Gd}_{2} \mathrm{O}_{3}$ would require a ternary mixture of $\mathrm{ThO}_{2}, \mathrm{PuO}_{2}$, and $\mathrm{Gd}_{2} \mathrm{O}_{3}$ which is very difficult to fabricate with good homogeneity. The IFBA concept entails a thin layer of zirconium boride applied to the surface of the fuel pellets [11].

In order to achieve an even power profile within the fuel assembly, three different levels of Pu content are used. The rods with the highest $\mathrm{Pu}$ content contain $13.7 \% \mathrm{Pu}$, the lowest $9 \% \mathrm{Pu}$, and the rest $11.7 \% \mathrm{Pu}$. The fuel assembly design is shown in Figure 4.

The mixture of $\mathrm{ThO}_{2}$ and $\mathrm{PuO}_{2}$ has a slightly lower density than $\mathrm{UO}_{2}$ due to the lower mass number of Th. Given 


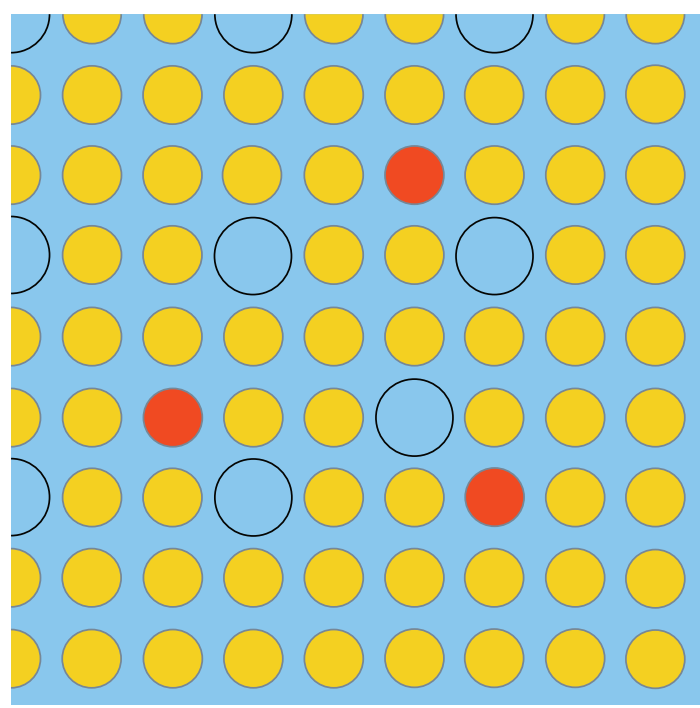

FIGURE 3: The BA-containing UOX reference fuel assembly design (a quarter of the fuel assembly). Yellow rods consist of $4.4 \%$ enriched uranium; red rods consist of $2.8 \%$ enriched uranium and $6 \% \mathrm{Gd}_{2} \mathrm{O}_{3}$. The non-BA-containing reference fuel design is identical except that all rods are of the former (yellow) type.

that the same mechanical structure is used, this results in a slightly lower assembly heavy metal weight; $431 \mathrm{~kg}$, assuming the same porosity.

\section{Method}

3.1. Simulation Tools. The fuel assembly simulations are carried out in two dimensions, using the fuel assembly burnup simulation program CASMO-4E [8] together with the microscopic cross-section library JEF2.2. CASMO-4E generates cross-section data for the subsequent use in core simulations.

The core simulations are carried out in three dimensions, using SIMULATE-3 [9] and the macroscopic crosssection libraries generated by CASMO-4E and converted by the linking program CMS-link (Studsvik Scandpower's Core Management System (CMS) linking program [12]). SIMULATE-3 is a two-group steady-state nodal code. All the used softwares are supplied by Studsvik Scandpower, Inc.

3.2. Reload Safety Evaluation. The Swedish Radiation Safety Authority requires that an RSE is performed for every core reload, in order to ensure that the reactor can be operated safely with the new core design during the whole cycle. The reload safety evaluation employed at Ringhals 3 is based on the Westinghouse reload methodology which is licensed by the United States Nuclear Regulatory Commission (USNRC) as described in the topical report WCAP-9272 [13]. This is a bounding analysis approach, and it is employed in this work in the same way as is usually done when a new reload is designed for Ringhals 3.

In the RSE, a number of key parameters are calculated. These are effective delayed neutron fraction $\beta_{\text {eff }}$, boron worth,

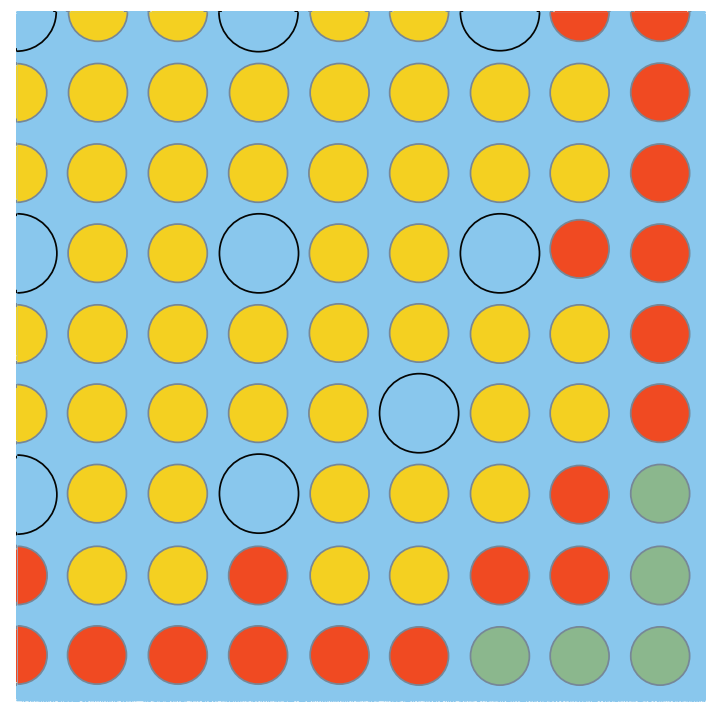

FIgure 4: The Th-MOX fuel assembly design (a quarter of the fuel assembly). Yellow rods contain $13.7 \%$, red rods $11.7 \%$, and grey rods $9.0 \% \mathrm{Pu}$ (weight percent of heavy metal content). The zirconium boride layer is too thin to be visible in the figure.

Shut Down Margins (SDM), Moderator Density Coefficient (MDC), Moderator Temperature Coefficient (MTC), Isothermal Temperature Coefficient (ITC), Doppler Temperature Coefficient (DTC), and Doppler Power Coefficient (DPC). For each of these (except for the MTC, in the case of Ringhals 3), there are a lower and/or upper safety limits, specific to the reactor for which the calculations are done. These safety limits take into account the uncertainty in the calculations.

The calculated values of key parameters are then used for checking whether safety conditions are fulfilled for a large number of transients; that is, whether there is departure from nucleate boiling and whether the burnup-dependent limit on the linear heat generation rate (LHGR) is contained.

\section{Results}

4.1. Depletion Behaviour. The infinite multiplication factor $k_{\infty}$ for the different fuel assemblies is shown in Figure 5. One clear difference between the Th-MOX and the reference fuel is that the reactivity decreases more slowly in the ThMOX case. Another difference is seen during the first cycle (approximately the first $1 / 3$ of the curves), and that is the difference in the rate with which the BA burns out. Whereas the $\mathrm{Gd}_{2} \mathrm{O}_{3}$ burns out fairly rapidly and almost linearly, the effect of the IFBA in the Th-MOX assembly lingers for a longer time and decays rather exponentially. In both cases, the objective of avoiding an exceedingly high $k_{\infty}$ at the Beginning Of Life (BOL) is fulfilled. If BA would not be used, the much higher reactivity of the fresh fuel assemblies compared with the once or twice burnt would cause locally high-power levels where the fresh fuel is located, which would limit the power at which the reactor can be safely operated. 


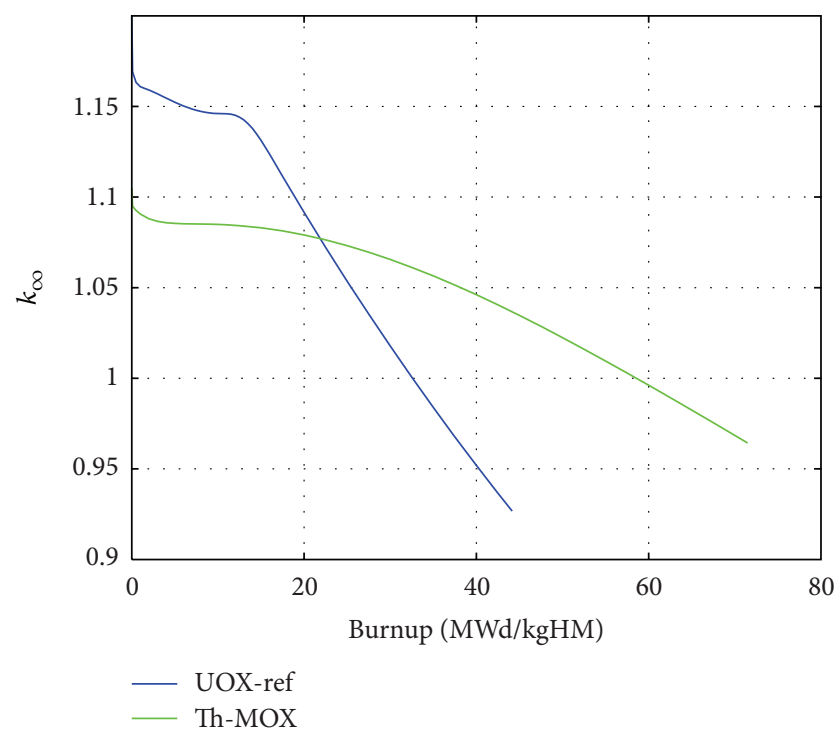

FIGURE 5: Infinite multiplication factor dependence on burnup.

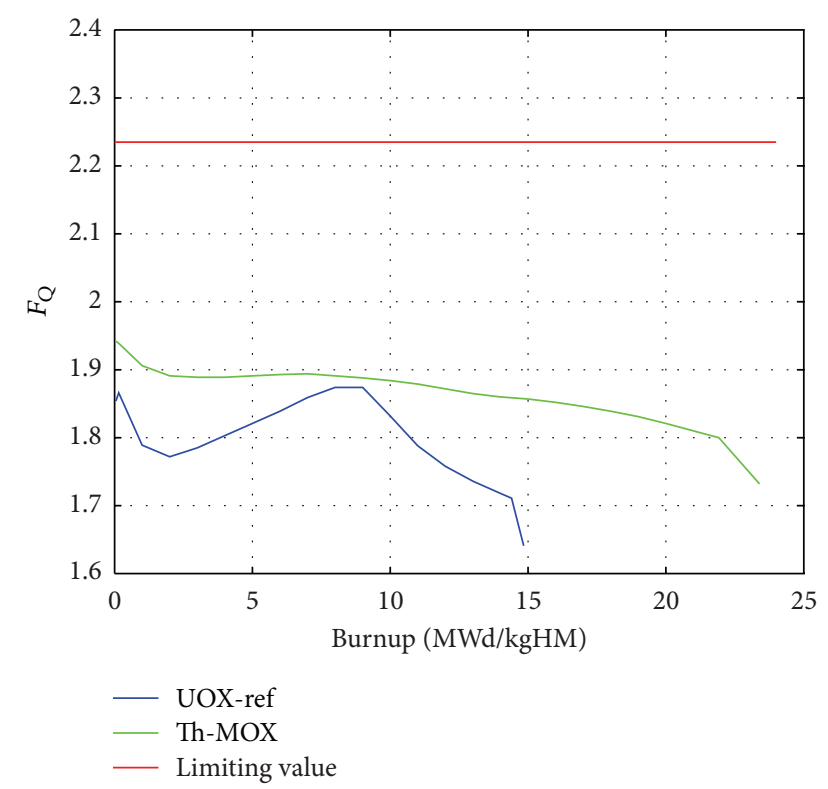

FIGURE 6: $F_{Q}$, that is, local power relative to core average power as a function of burnup.

Also, a higher concentration of soluble boron would be needed to keep the reactor critical.

In order to reach the desired operating time of three 18-month cycles, the assembly average discharge burnup is increased to $78 \mathrm{MWd} / \mathrm{kgHM}$ in the Th-MOX case. It should be noted that this discharge burnup is considerably higher than the currently employed limits on UOX fuel. There are several reasons to believe that this burnup will in fact be achievable. Firstly, although the burnup is high, the residence time within the reactor is only 4.5 years. Whereas this residence time is quite long for PWR fuel, it is a normal residence time for boiling water reactor fuel, so fuel aging factors related to the residence time should be within acceptable range.
Secondly, it is expected that thorium-based fuel will have a capacity to keep its thermal and mechanical robustness to high burnup, for several reasons. One is that the thermal conductivity of the Thorium-Plutonium Mixed Oxide ceramic is higher than that of a corresponding uranium oxide ceramic, up to about $14 \% \mathrm{Pu}[6]$. This means that the fuel temperature will remain lower in the pellet, causing less swelling, less pellet-cladding mechanical interaction, and a larger margin to fuel melting. Another reason is that fission gases have a lower mobility in the thorium oxide matrix, which results in a lower fission gas release. The higher thermal conductivity also has a beneficial effect on the fission gas release, since the lower fuel temperature makes the fission gases diffuse more slowly.

Deterioration of the cladding integrity may constitute an obstacle to safe operation at high burnups. This is partially mitigated by the fact that the residence time is not extremely long, and there are documented cases of cladding operating well up to such burnups [14]. There are also interesting initiatives towards combining thorium fuel with silicon carbide cladding for realizing much higher burnups than the current standard [15].

4.2. Power Distribution. As mentioned, the development of the infinite multiplication factor with burnup is important for maintaining an even power distribution within the core. The parameter $F_{\mathrm{Q}}$ denotes the maximum local fuel rod linear power density divided by the average fuel rod linear power density.

$F_{\mathrm{Q}}$ is calculated for equilibrium xenon at Beginning $\mathrm{Of}$ Cycle (BOC), Middle Of Cycle (MOC), End Of Gadolinium (EOG), and at End Of Full Power (EOFP). The highest $F_{Q}$ in the core, for normal operating conditions, is plotted in Figure 6 as a function of burnup. As can be seen, $F_{Q}$ is higher for the Th-MOX fuel than for the reference fuel but stays comfortably below the limiting value.

Another measure of the evenness of the power distribution is $F_{\Delta H}$, which for each fuel rod denotes the integral rod power of that fuel rod relative to the average integral rod power. This parameter is calculated similarly to $F_{Q}$ and the highest value, corresponding to the hottest rod, is plotted in Figure 7. In this case, the value for Th-MOX is similar and even a bit lower than that for the reference fuel, and also below the limiting value.

The limits are set by the material properties of the fuel, and the same limits are employed for the Th-MOX core and the reference core. The limits displayed in these plots are the least restrictive ones, which are applicable for fresh fuel. For older fuel, lower limits are applied. A more detailed analysis shows that some of the older fuel assemblies break the applicable age-dependent limits in the Th-MOX case. However, as discussed in Section 4.1, there are reasons to believe that Th-MOX fuel will have better material properties than UOX fuel also at high burnup.

Keeping the power distribution even has two main purposes, one being economical utilization of the fuel and the other one being to allow operation at high power without reaching departure from nucleate boiling anywhere 


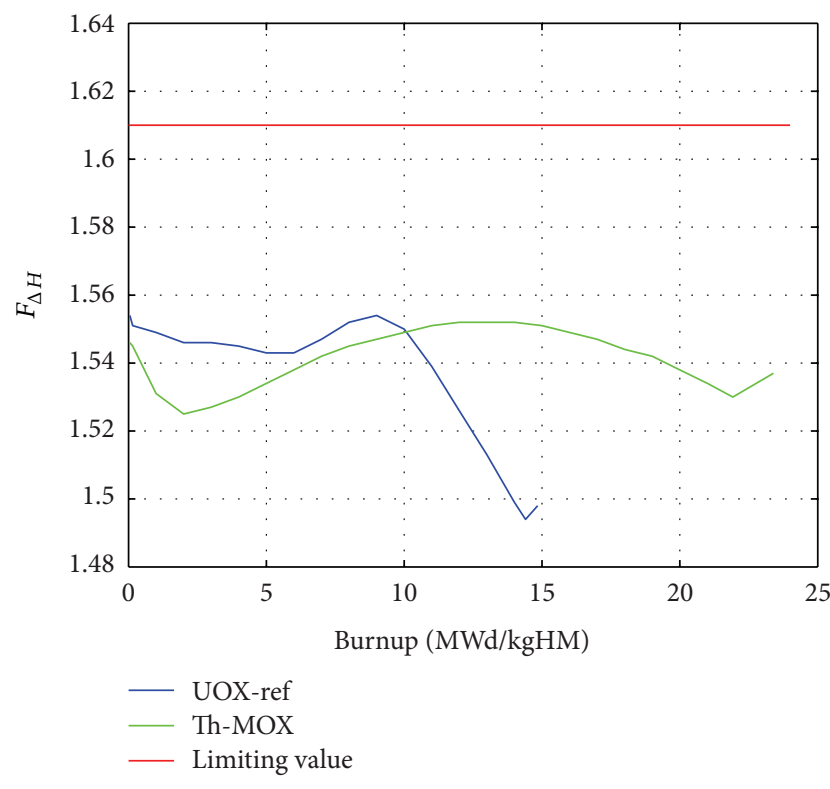

FIGURE 7: $F_{\Delta H}$, that is, maximum rod power relative to core average rod power as a function of burnup.

in the core. A measure of how large the margin is to departure from nucleate boiling is the Departure from Nucleate Boiling Ratio (DNBR). This is the ratio between the heat flux which would cause departure from nucleate boiling and the actual local heat flux at a fuel rod surface and should thus be kept above unity, and preferably above a certain threshold to provide margin to departure from nucleate boiling in the case of transient events. The threshold employed in Ringhals 3 is 1.36 . The reference fuel is very slightly above the limit, whereas the Th-MOX fuel has a larger margin with a cycle minimum DNBR of 1.43. This is a very positive feature of the core, which provides extra margin in a large number of transients.

4.3. Control Systems. The boron worth is calculated for boron concentrations between 0 and $2500 \mathrm{ppm}$ and for the whole power range, from Hot Zero Power (HZP) with no xenon to Hot Full Power (HFP) with xenon equilibrium. Usually natural boron is used for reactivity control, that is, with $19.8 \%{ }^{10} \mathrm{~B}$, which is the isotope having the largest thermal neutron capture cross section. Using this isotopic composition, the boron worth is significantly reduced (closer to zero) in the Th-MOX case. This is expected, since the presence of large absorption resonances in several of the $\mathrm{Pu}$ isotopes causes the flux to decrease at the thermal energies where ${ }^{10} \mathrm{~B}$ has its highest absorption cross section. In order to cope with this problem, the Th-MOX cycle is simulated with boron enriched to $60 \%$ in ${ }^{10} \mathrm{~B}$. As can be seen in Table 1 , this gives a boron worth for the Th-MOX core similar to that in the reference UOX core, and within the safety limits.

The flatter slope of $k_{\infty}$, which allows for a lower initial $k_{\infty}$ of the Th-MOX assembly, lowers the boron concentration needed for keeping $k_{\text {eff }}$ equal to unity. The use of enriched

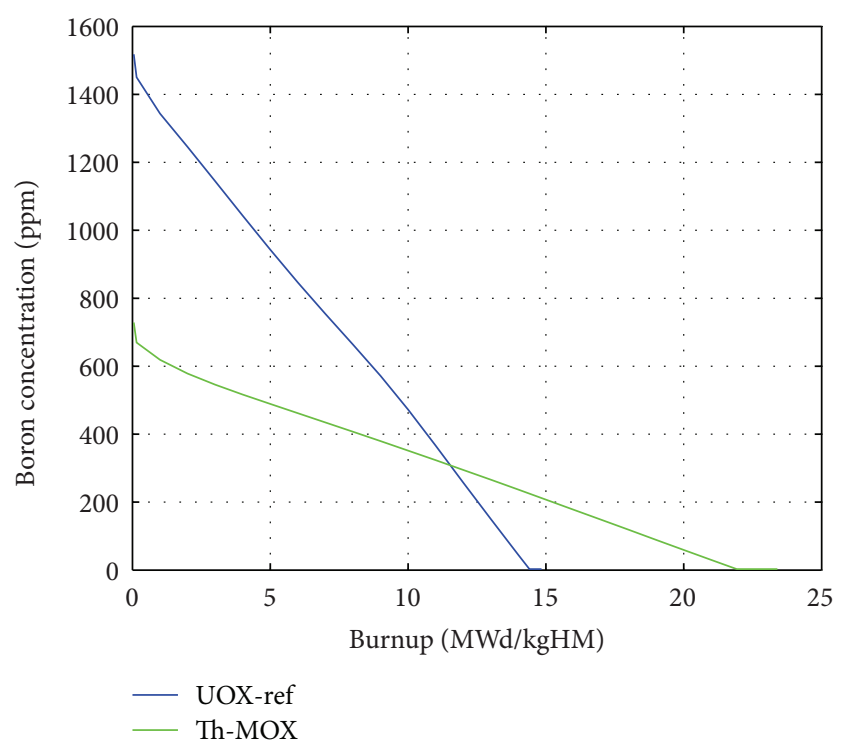

FIGURE 8: Boron concentration as a function of burnup.

TABLe 1: Control rod and boron worth, their limits, and the calculated values for the Th-MOX and the reference UOX core. For the Th-MOX core, the boron used for reactivity control is enriched to $60 \%$ in ${ }^{10} \mathrm{~B}$ instead of the usual $19.8 \%$ (natural isotopic composition), and stronger control rods are used.

\begin{tabular}{lccc}
\hline Parameter & Limit & UOX-ref & Th-MOX \\
\hline Min boron worth $[\mathrm{pcm} / \mathrm{ppm}]$ & -15 & -8.3 & -8.7 \\
Max boron worth $[\mathrm{pcm} / \mathrm{ppm}]$ & -5 & -6.2 & -5.2 \\
Min SDM [pcm] & 2000 & 2559 & 3895 \\
Max rod worth [pcm] & - & 6505 & 7808 \\
\hline
\end{tabular}

boron enhances this effect significantly, and the result is shown in Figure 8.

The same mechanisms that cause the lower boron worth for Th-MOX fuel also cause a significant lowering of the control rod worth. In order to adhere to the limits on $\mathrm{SDM}$, the normal control rods, containing a silver-indiumcadmium alloy as the neutron absorber, had to be exchanged for stronger ones containing $\mathrm{B}_{4} \mathrm{C}$. This shortens the lifetime of the control rods but improves the shutdown margin significantly. Furthermore, the four extra openings available in the Ringhals 3 pressure vessel have been utilized for inserting four extra control rods in addition to the 48 which are normally used. The values shown in Table 1 show that the minimum SDM with the $\mathrm{B}_{4} \mathrm{C}$ control rods is well above the lower limit.

Also the maximum control rod worth is shown in Table 1 and proves to be higher than the reference. This gives a higher value of the Ejected Rod Worth (ERW) in case of a Rod Ejection Accident (REA) when this transient is evaluated at HZP. However, when evaluated at HFP, the ERW is smaller than the reference, as discussed in Section 4.5. This is most likely due to the strong DTC, discussed in Section 4.4. The same tendency can be seen for the Differential Rod Worth 
TABLE 2: Key safety parameters, their limits, and the calculated values for the Th-MOX and the reference UOX core. No limit is specified for moderator temperature coefficient in Ringhals 3.

\begin{tabular}{lccc}
\hline Parameter & Limit & UOX-ref & Th-MOX \\
\hline Max MDC & 0.50 & 0.41 & 0.34 \\
{$\left[(\Delta k / k) /\left(\mathrm{g} / \mathrm{cm}^{3}\right)\right]$} & - & -1.3 & -4.0 \\
$\operatorname{Max}$ MTC $[\mathrm{pcm} / \mathrm{K}]$ & 0.0 & -4.4 & -7.9 \\
Max ITC $[\mathrm{pcm} / \mathrm{K}]$ & -4.00 & -3.72 & -4.53 \\
$\operatorname{Min}$ DTC $[\mathrm{pcm} / \mathrm{K}]$ & -1.70 & -2.10 & -2.36 \\
Max DTC $[\mathrm{pcm} / \mathrm{K}]$ & -21.0 & -15.5 & -21.5 \\
$\operatorname{Min}$ DPC $[\mathrm{pcm} / \%$ power $]$ & -6.5 & -9.8 & -14.6 \\
Max DPC $[\mathrm{pcm} / \%$ power $]$ & 430 & 502 & 350 \\
Min $\beta_{\text {eff }}$ EOC $[\mathrm{pcm}]$ & 720 & 647 & 370 \\
Max $\beta_{\text {eff }}$ BOC $[\mathrm{pcm}]$ & & &
\end{tabular}

(DRW) in case of Rod Withdrawal At Power (RWAP), also discussed in Section 4.5.

4.4. Key Safety Parameters. The MDC quantifies the reactivity change caused by a change in moderator density when all other parameters, such as core power, moderator flow, and fuel temperature, are kept constant. The highest and hence most limiting MDC occurs at End Of Cycle (EOC), HFP, All control Rods Inserted (ARI), a boron concentration of $0 \mathrm{ppm}$, and the highest average moderator temperature, $305.3^{\circ} \mathrm{C}$. This is clearly not a set of authentic operating parameters; for example, the core is never operated at HFP with ARI, but it guarantees that the calculated value of MDC is conservative. As can be seen in Table 2 the MDC of the Th-MOX core is smaller than that of the reference core and is well within the safety limits. As will be discussed below, this small value of the MDC is beneficial in many transient scenarios. Many transients involve a cooling of the core, which leads to an increase of the moderator density. The small positive MDC in the Th-MOX core means that the associated reactivity increase is comparatively small.

A high MDC corresponds to a low MTC and vice versa, so the highest MTC occurs at the operating conditions corresponding to the lowest MDC, which are BOC, HZP, All control Rods Out (ARO), no xenon, and critical boron concentration. The MTC is the ratio between the reactivity change caused by a change in moderator temperature, divided by the magnitude of that temperature change. For Ringhals 3 , a limit is defined for the ITC instead of the MTC although the MTC is used in many transient simulations and is thus of great interest. The MTC at BOC for Th-MOX fuel is significantly lower than that of the reference, providing a larger margin to becoming positive. Later in the cycle, however, the MTC of the Th-MOX fuelled core is higher than that of the reference core $(-75 \mathrm{pcm} / \mathrm{K}$ at EOC to be compared with $-89 \mathrm{pcm} / \mathrm{K}$ for the reference). This means, once again, that many transients are handled in a better way, since the reactivity increase related to a temperature decrease is smaller for Th-MOX fuel than for the reference UOX fuel.

The ITC corresponds to the change in reactivity caused by a simultaneous increase of the temperature of all present materials while all other parameters are kept constant. The limiting ITC occurs at BOC, HZP, no xenon, and maximum samarium. The ITC for both the Th-MOX and the reference core is well within the safety limits, the ITC for the Th-MOX core being significantly larger in amplitude than that of the reference core.

The MDC, MTC, and ITC all show the same tendency of larger amplitudes for the Th-MOX fuel than for the reference. This is primarily due to the two competing effects of a moderator density increase (associated with a temperature decrease in the MTC and ITC cases). An increase in the moderator density causes an increase in the parasitic neutron absorption by the moderator. This effect is independent on fuel type. However, the density increase also improves the moderation which causes a thermalization of the neutron spectrum. This in turn increases the macroscopic fission cross section to an extent which depends on the specific energy dependence of the microscopic fission cross sections of the fissile nuclei present in the fuel. In the Th-MOX fuel, the dominating fissile isotopes are ${ }^{239} \mathrm{Pu}$ and ${ }^{241} \mathrm{Pu}$, which have large peaks in their microscopic fission cross sections at about $0.3 \mathrm{eV}$. The main fissile isotope in the reference UOX fuel is ${ }^{235} \mathrm{U}$, which has a smaller peak at $0.3 \mathrm{eV}$. Pu bearing fuels thus benefit more than UOX fuel from the thermalization of the neutron spectrum caused by a density increase.

The DTC indicates the reactivity response caused by a change in fuel temperature only, due to the Doppler broadening of the absorption resonances of the fuel material. This parameter varies quite irregularly with power and burnup, so it is calculated for several different sets of operating conditions and a minimum and maximum value is sought. Due to the presence of several nuclides in the Th-MOX fuel, the DTC is lower (larger in amplitude, negative) in the ThMOX core than in the reference UOX core, below the lower safety limit. When the result is outside the safety limits, a closer investigation must be made of the transient simulations where the minimum value of the DTC is used. These are Feed Water Malfunction (FWM), Steam Line Break (SLB), at HFP and HZP, Feed Line Break (FLB), Inadvertent Safety Injection (ISI) and the previously mentioned REA and RWAP transients. These are the same transient simulations that use the minimum value of $\beta_{\text {eff }}$ and will be discussed in the following section.

A closely related parameter is the DPC. It is defined as the reactivity change due to a change in core power, which is related to a corresponding change in fuel and cladding temperatures while the moderator temperature is fixed. The physical principle behind the DPC is thus the same as for the DTC. The DPC is also calculated at a large number of burnup and power levels. For illustration, the DPC at $100 \%$ power is listed in Table 2, since most transients start at this power level. As can be seen, the DPC is slightly below the lower safety limit in the Th-MOX fuelled core. The transients where the minimum DPC is used are the following: Loss Of Normal Feedwater/Offsite Power (LONF/LOOP), Partial/Complete Loss of Flow (PLOF/CLOF), Locked Rotor (LR), and Steam Generator Tube Rupture (SGTR) and also some of the same transients that use the minimum DTC: FLB, RWAP, and ISI. Also these transients will be discussed in Section 4.5. 
TABLE 3: Calculated safety parameters for the representative transients SLB, REA, and RWAP. For REA, the parameters and limits are for EOC conditions. For SLB and RWAP, the same limits are employed throughout the cycle and the parameters which are listed for the moment in the cycle where they are closest to these limits.

\begin{tabular}{lccc}
\hline Parameter & Limit & UOX-ref & Th-MOX \\
\hline $\begin{array}{l}\text { SLB at HZP, limiting moment } \\
\quad\end{array}$ & 2.93 & 4.56 & 5.37 \\
$\quad$ Min DNBR [-] & & & \\
$\begin{array}{l}\text { SLB at HFP, limiting moment } \\
\quad \text { Min DNBR [-] }\end{array}$ & 1.36 & 1.86 & 1.93 \\
$\quad$ Max LHGR [W/cm] & 545 & 426 & 440 \\
REA at HZP, EOC & & & \\
$\quad$ Max ERW [\$] & 2.55 & 0.99 & 1.12 \\
$\quad$ Max FQ [-] & 59.2 & 15.3 & 9.48 \\
REA at HFP, EOC & & & \\
$\quad$ Max ERW [\$] & & & \\
$\quad$ Max F [-] & 0.30 & 0.056 & 0.047 \\
RWAP at HFP, limiting moment & & & 2.183 \\
$\quad$ Max DRW [pcm/cm] & 50 & 11.95 & 10.88 \\
\hline
\end{tabular}

The effective delayed neutron fraction $\beta_{\text {eff }}$ is calculated at BOC and EOC for HZP, ARO, no xenon, and maximum samarium. $\beta_{\text {eff }}$ is strongly affected by the fissioning isotope. The numbers in Table 2 mainly reflect the fact that the delayed neutron fraction is considerably lower (less than half) in ${ }^{239} \mathrm{Pu}$ and ${ }^{233} \mathrm{U}$ compared with ${ }^{235} \mathrm{U}$, which leads to a $\beta_{\text {eff }}$ below the lower safety limit. This indicates that the ThMOX-core will have a smaller margin to prompt criticality compared with the reference. As mentioned, the minimum value of $\beta_{\text {eff }}$ is used in the same transients as the minimum DTC: FWM, SLB at HZP and HFP, FLB, RWAP, ISI, and REA. These transients are discussed below.

4.5. Transient Simulations. A large number of transients have previously been simulated in large detail for the accepted values of the safety parameters, that is, for the values between the upper and lower limits shown in Table 2. As long as the key parameters fall within the safety limits, these general simulations are applicable and the outcome of the transient is known to be acceptable. When any of the key parameters discussed above falls outside the safety limits, the affected transients must be checked in order to determine whether the outcome is acceptable. As noted above, $\beta_{\text {eff }}$, DPC, and DTC fall outside these safety limits for Th-MOX fuel, which means that the transient simulations which have been carried out for Ringhals 3 have not been carried out for the calculated values for these parameters. A discussion of the affected transients follows, indicating why they are likely to be acceptable even though the simulation results are not exactly applicable. Some representative simulation results are listed in Table 3 , showing that the margins are large in all cases, and in many cases larger for Th-MOX than for the reference fuel.

All of the transients where the minimum value of $\beta_{\text {eff }}$ is used, except for REA, are rather slow transients; that is, they happen over a time scale of a few seconds. Within this time frame, there is time for the comparatively slow feedback process provided by the MTC and the MDC to contribute.

In the cases of FWM, SLB, and FLB, the moderator temperature sinks and the density increases. The generally weaker reactivity feedback provided by the higher MTC and lower MDC in the Th-MOX case makes the consequent reactivity increase smaller compared with the reference case, which causes the Th-MOX core to behave well in these transients despite the lower $\beta_{\text {eff }}$. DNBR and in the HFP case also LHGR are checked and the calculated values for SLB are listed in Table 3 . As previously mentioned, the margins to the limiting values are seen to be large. There are small differences between Th-MOX and the reference, which depend partly on the fuel type and partly on the core design in each case.

An ISI involves an increased flow of borated water to the core. This transient has large margins and the power never increases above $100 \%$, neither for the Th-MOX core nor the reference core, so this transient does not imply any safety concerns.

For the REA transient, the minimum value of $\beta_{\text {eff }}$ is of larger importance, since the transient is fast. However, the results of the analysis show that all investigated parameters (the maximum ejected rod worth and $F_{\mathrm{Q}}$ ) were within the employed limits with good margins throughout the transient. The reason for this is that the large negative DPC and DTC of the Th-MOX core provide strong and rapid negative reactivity feedback. In the HFP case, where the fuel temperature is high, the ERW is even lower for the Th-MOX fuel than for the reference. Given the large margins there is good reason to believe that a Th-MOX-fuelled core would cope well with this transient even though the actual value of $\beta_{\text {eff }}$ is smaller than the one used in the simulations.

RWAP is similar to REA but happens more slowly, so the large negative DPC should make also this transient acceptable. The listed value of the maximum differential rod worth for the Th-MOX case, showing an even larger margin to the limit than the reference, strengthens this assumption.

As mentioned above, the transients in which the minimum DPC plays a role also involve the value of $\beta_{\text {eff }}$, but the maximum value of $\beta_{\text {eff }}$ rather than the minimum value discussed above. The main concern in these cases is the reactivity increase after a SCRAM caused by the low DPC. However, the strong (and added) control rods provide an extra reactivity margin during and after a SCRAM, which makes it very likely that also these transients will turn out well. Also, the small value of the maximum $\beta_{\text {eff }}$ makes the power decrease at SCRAM come faster than in the reference case, which leaves a smaller decay power that needs to be cooled off.

\section{Conclusions}

The simulations, which have been carried out according to the same routines which are normally employed when designing a new reload, do not show any fundamental obstacles to loading the Ringhals 3 core with Th-MOX fuel with maintained safety margins in normal operation and transient scenarios. However, the condition is that the control 
systems are upgraded: Boron enriched to $60 \%$ in ${ }^{10} \mathrm{~B}$ must be used for reactivity control, and stronger control rods than the current ones must be used for shutdown. In our simulations, control rods with $\mathrm{B}_{4} \mathrm{C}$ are used, which fulfil the safety conditions. In addition, it might be necessary to utilize the four currently unused control rod openings for inserting four additional control rods.

Three kinetic parameters, namely, the minimum Doppler power, temperature coefficients, and the minimum effective delayed neutron fraction $\beta_{\text {eff }}$, have values outside the current safety limits. However, differences in the values of the reactivity coefficients along with the extra margins at SCRAM provided by the use of stronger control rods make it possible to argue that the Th-MOX-fuelled core will behave acceptably also in transients where these parameters are of importance.

What remains to be done is to carry out the transient simulations in detail, using the exact calculated values of the key parameters for Th-MOX fuel. This is a major undertaking, which does not only require a large amount of computing capacity and modelling work, but also that temperature and power limits specific to Th-MOX fuel are determined experimentally. These parameters are assessed in an upcoming experiment which will be carried out in the Halden research reactor [16]. This experiment also aims to experimentally confirm the claims made about Th-MOX fuel being able to withstand higher burnup than UOX fuel while maintaining acceptable thermal and mechanical robustness.

Ringhals 3 is a typical PWR, which started commercial power production as early as 1981 . Hence, it has no features which would make it particularly suited for using Th-MOX fuel. Thus, our conclusion, based on this limited set of simulations, is that PWRs in general can be loaded with ThMOX fuel, given that the control systems are strong enough and that the properties of the Th-MOX fuel material become experimentally determined.

\section{Acknowledgments}

The authors would like to thank Ringhals AB for the scientific and technical support and the Norwegian Research Council, Vattenfall AB, Oskarshamns Kraftgrupp AB, and the Swedish Centre for Nuclear Technology for the financial support. The support from the Norwegian Research Council was provided through the industrial Ph.D. program.

\section{References}

[1] H. R. Trellue, C. G. Bathke, and P. Sadasivan, "Neutronics and material attractiveness for PWR thorium systems using monte carlo techniques," Progress in Nuclear Energy, vol. 53, no. 6, pp. 698-707, 2011.

[2] E. Fridman and S. Kliem, "Pu recycling in a full Th-MOX PWR core-part I: steady state analysis," Nuclear Engineering and Design, vol. 241, no. 1, pp. 193-202, 2011.

[3] H. Tsige-Tamirat, "Neutronics assessment of the use of thorium fuels in current pressurized water reactors," Progress in Nuclear Energy, vol. 53, no. 6, pp. 717-721, 2011.

[4] K. Insulander Björk, V. Fhager, and C. Demazière, "Comparison of thorium-based fuels with different fissile components in existing boiling water reactors," Progress in Nuclear Energy, vol. 53, no. 6, pp. 618-625, 2011.

[5] M. Todosow and G. Raitses, "Thorium based fuel cycle options for PWRs," in Proceedings of the International Congress on Advances in Nuclear Power Plants (ICAPP '10), pp. 1888-1897, San Diego, Calif, USA, June 2010.

[6] C. Cozzo, D. Staicu, J. Somers, A. Fernandez, and R. Konings, "Thermal diffusivity and conductivity of thorium-plutonium mixed oxides," Journal of Nuclear Materials, vol. 416, no. 1-2, pp. 135-141, 2011.

[7] M. Karam, F. Dimayuga, and J. Montin, "Fission gas release of (Th, $\mathrm{Pu})_{2} \mathrm{O}_{2}$ CANDU fuel,” Tech. Rep. CW-124950-CONF-002, Atomic Energy of Canada Limited, 2008.

[8] J. Rhodes, D. Lee, and K. Smith, CASMO-4E: Extended Capability CASMO-4-USer'S Manual, Studsvik Scandpower, 2009.

[9] D. W. Dean, SIMULATE-3 Advanced Three-Dimensional TwoGroup Reactor Analysis Code, Studsvik Scandpower, 2007.

[10] "Plutonium," World Nuclear Association, 2009, http://www .world-nuclear.org/info/inf15.html.

[11] A. Biancheria and R. J. Allio, "Nuclear fuel elements," US patent 3.427.111, 1969.

[12] T. Bahadir, CMS-LINK USer's Manual, Studsvik Scandpower, 1999.

[13] "Westinghouse reload evaluation methodology," Tech. Rep. WCAP-9272, US Nuclear Regulatory Commission, 1978.

[14] G. Ledergerber, S. Valizadeh, J. Wright et al., "Fuel performance beyond design-exploring the limits," in Proceedings of the LWR Fuel Performance Meeting/Top Fuel/WRFPM, pp. 513-524, Orlando, Fla, USA, September 2010.

[15] E. Shwageraus and H. Feinroth, "Potential of silicon carbide cladding to extend burnup of $\mathrm{Pu}$-Thmixed oxide fuel," Transactions of the American Nuclear Society, vol. 104, pp. 658-660, 2011.

[16] J. F. Kelly and V. Fhager, "Designing a thorium fuel irradiation experiment," in Advanced Fuel Pellet Materials and Fuel Rod Design for Water Cooled Reactors, no. TECDOC 1654, International Atomic Energy Agency, 2010. 


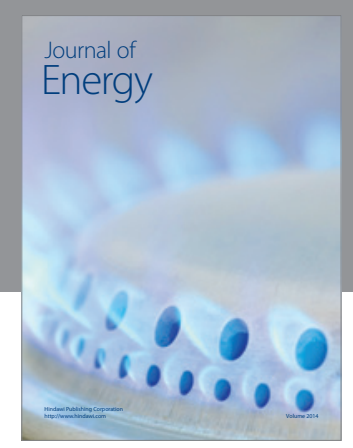

Journal of

Industrial Engineering
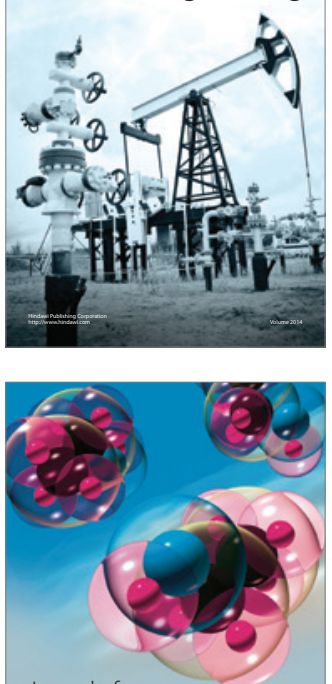

Fuels
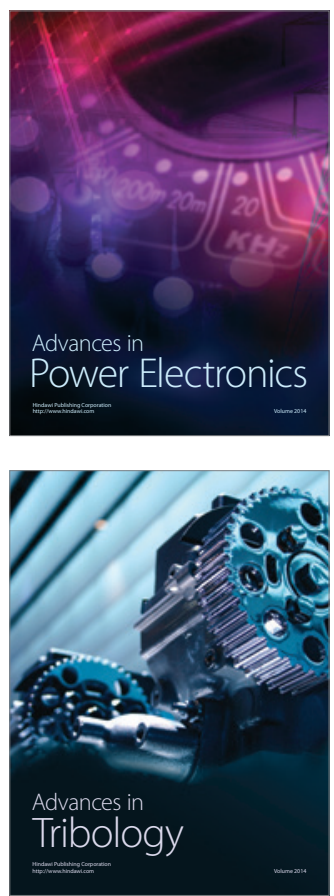

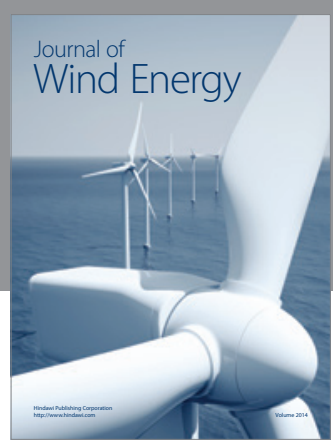

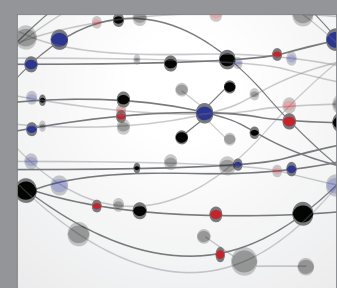

The Scientific World Journal

Submit your manuscripts at http://www.hindawi.com

Journal of

Structures
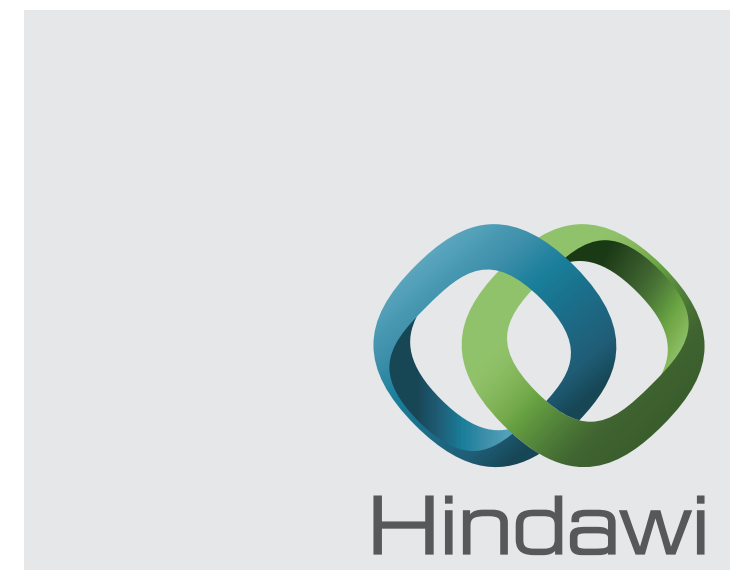

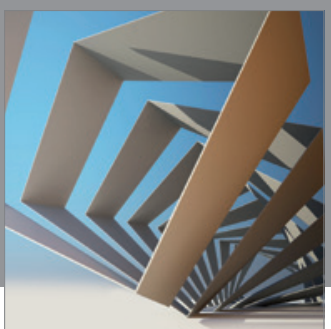

Rotating

Machinery
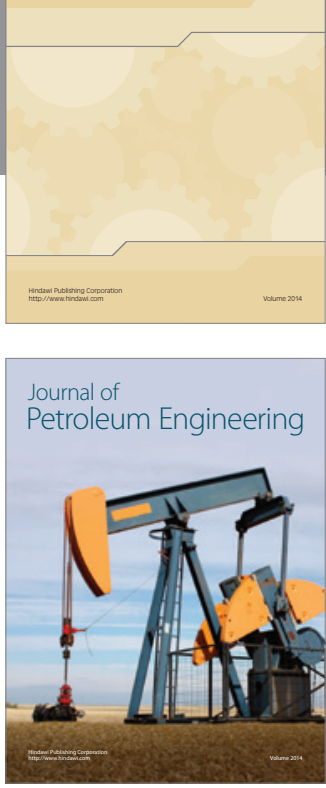

Journal of

Solar Energy
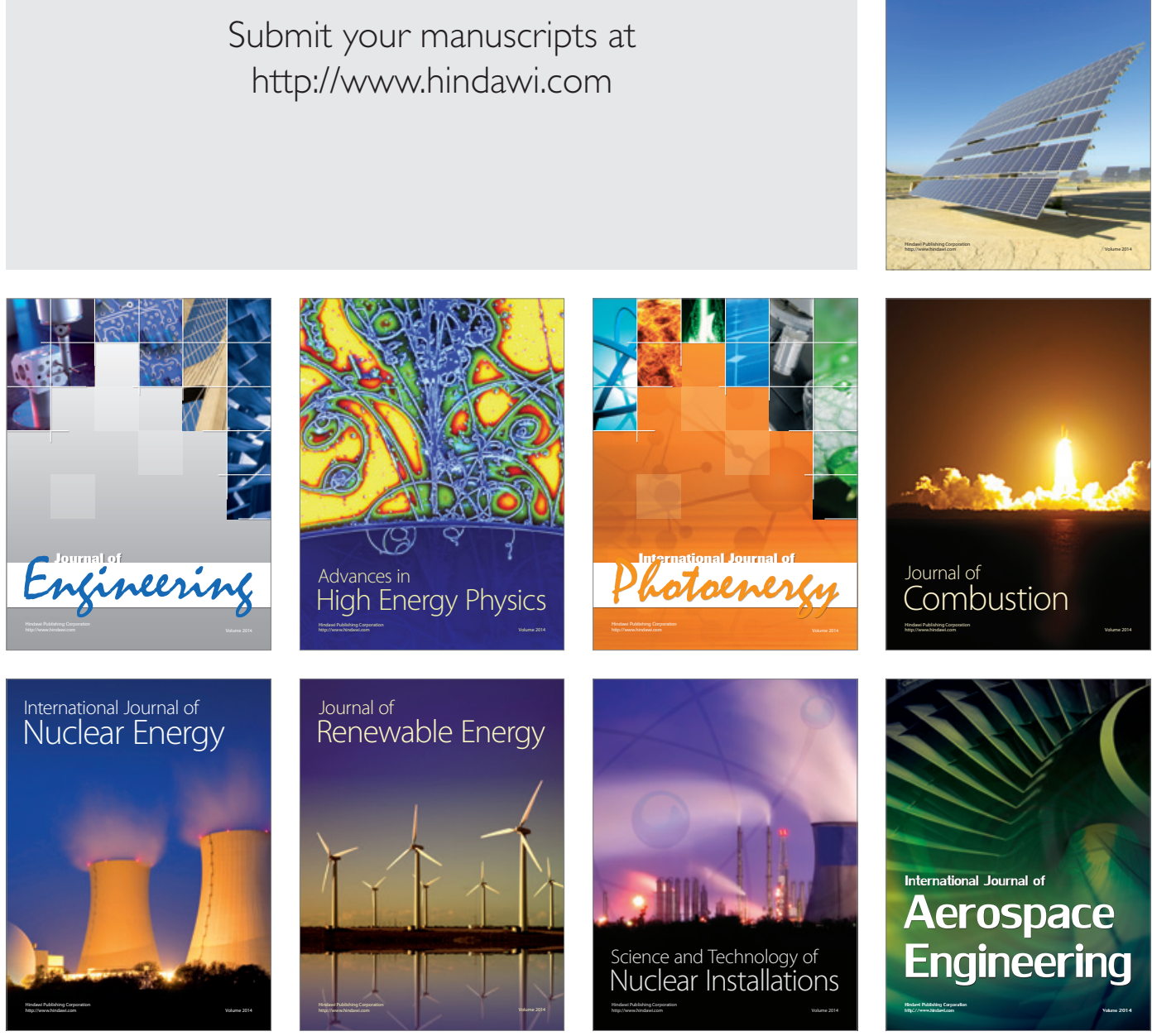